\title{
Investigation of the Simultaneous Volumetric 3-component Flow Field inside a Hydrocyclone
}

\author{
Jiangang Wang, Zhishan Bai, Qiang Yang, Yi Fan, Hualin Wang* \\ ${ }^{a}$ State Environmental Protection Key Laboratory of Environmental Risk Assessment and \\ Control on Chemical Process, East China University of Science and Technology, Shanghai, \\ 200237, PR China
}

\begin{abstract}
The separation mechanism of hydrocyclone is elucidated by determining the three velocity components of the flow field. In this study, volumetric 3-component velocimetry measurement system was developed to investigate the three-dimensional three-component flow field inside a $35 \mathrm{~mm}$ mini-hydrocyclone. Measurement results show the magnitude of maximum tangential, axial, radial velocity component in r-z plane of the cylindrical and cone part is about $4: 2: 1$. The parameter $n$ of tangential velocity in the free vortex area is fitted to be 0.5-0.7, which is a variable along axial direction. Radial velocity is non-axisymmetric, while the other two are quasi - axisymmetric. The asymmetry of the radial velocity is found to be the consequence of the existing downward progression of secondary vortices. In addition, the locus of vertical velocity was constructed in three dimensional and the shortcut flow rate was calculated using three dimensional axial velocity. These knowledge helps the further

${ }^{*}$ Corresponding author: Environmental Protection Key Laboratory of Environmental Risk Assessment and Control on Chemical Process, East China University of Science and Technology, 130 Meilong Road, Shanghai, 200237, PR China. Tel.:+86 21 64252748; Fax:+86 2164251894

E-mail address: samwhl@163.com (H.L.Wang).
\end{abstract}


understanding of the separation mechanism of the hydrocyclone.

Keywords: flow field; separation; three-dimensional three-component; secondary vortex; locus of vertical velocity

\section{Introduction}

Hydrocyclones are devices used to separate heterogeneous mixture in mineral processing [1], petrochemical [2], environmental [3] and other industries. The separation mechanism of hydrocyclone is elucidated by determining the three velocity components of the flow field. Tangential velocity determines the centrifugal separation factor for dispersed particles, whereas axial velocity, especially the zero axial velocity, defines the separation zone or space, and radial velocity affects the radial transportation behavior [4]. Some structures in hydrocyclonic flow field, including the locus of zero vertical velocity (LZVV), the shortcut flow and the circulation flow (secondary flow), also play an important role in the understanding of the separation mechanism.

\section{Table 1. Measurements on the flow field of hydrocyclone}

Up to recent years, worldwide researchers made great progresses on the measurement of hydrocyclone flow field, a summary of these measurements is shown in table 1 [5-21]. But the measurement of the three velocity components inside a hydrocyclone is still limited by the following aspects:

a. Radial velocity measurement: Radial component is the most difficult to measure in the flow field inside a hydrocyclone. Early studies failed to measure this component; thus, radial velocity was calculated depending on the material balance with the other 
two measured velocity components $[5,6]$. The calculation was based on the premise that the flow field was axisymmetric and the center of the fluid flow is consistent with the geometric center. Phase Doppler particle analyzer (PDPA) or laser Doppler velocimetry (LDV) were subsequently introduced in the measurement of the hydrocyclone flow field. Although PDPA is difficult to configure for measuring radial velocity, and the majority of previous reports using laser Doppler techniques did not include the radial velocity profile (see in table 1), several studies were able to elucidate some aspects of the radial velocity profile. Luo [8] and Chu [10] indicated that radial flow is exclusively inward in the cone section. By contrast, Fisher reported that radial velocity varies axially from inward to outward [11]. Marins [14] reported the radial velocity in the $1^{\circ}$ cone section. He reported an inward radial velocity near the wall and outward radial velocity near the axis. Overall, there still exists problems in measuring radial velocity using PDPA because the radial velocity may be non - axisymmetric when the measurement was based on axi-symmetry of the geometry.

b. Volumetric measurement of the 3D hydrocyclone flow field: Several studies reported the asymmetric feature of the flow field inside a hydrocyclone [22]. However, the 3D asymmetry in a flow volume was not measured because of the insufficiency of available methods. LDV/PDPA involves single-point measurement and time consuming. This characteristic forced studies to adopt axisymmetric characteristics for the three velocity components to minimize workload. A half $\mathrm{r}-\mathrm{z}$ plane was always measured to represent the entire flow field. However, these techniques are limited by the point-by-point 
measurement of the velocity components. Thus, these techniques are not appropriate for 3D flow measurement. PIV, which is as an imaging measurement method, is a promising alternative to LDV/PDPA. The PIV technique extends the measurement of hydrocyclone flow field from one point to two dimensions. However, PIV is still limited in the measurement of 3D flow and few PIV reports could be found in the measurement of hydrocyclone flow field.

c. Simultaneous and instantaneous measurements of the three velocity components: Bergstrom [4] found that most reports on tangential and axial velocities are not subjected to radial velocity. Moreover, instantaneous velocity measurement to specify the turbulence is also a difficult task.

These difficulties are caused by the limitation of available measurement methods. However, the development of imaging measurement methods has led to the formulation of three - dimensional three - component (3D3C) measurement techniques to solve the aforementioned limitations. Volumetric 3-component velocimetry (V3V) visualizes the entire field for flow field measurement [23-25]. The comparison of V3V with PIV and LDV/PDPA is shown in Table 2. V3V, developed based on the work of Pereira [26], combines defocusing technique and PIV/PTV technology. V3V technique provides an effective solution for velocity measurement of a highly instantaneous and 3D flow field.

\section{Table 2. Comparison of the three optical measurement methods}

The quality of particle image is important in image measurement methods [27]. The curved surface of a hydrocyclone adversely affects particle recognition. In previous reports of 
$\mathrm{V} 3 \mathrm{~V}$ measurements, the measurement was commonly conducted in open space without boundaries. However, for a flow field inside a hydrocyclone, the cylinder-on-cone-shaped hydrocyclone wall will greatly distort the particle image. These PIV measurements on the hydrocyclone flow did not present any index matching methods, suggesting great potential for improving velocity accuracy.

This study is the first to investigate the 3D3C flow field comprehensively by using V3V measurements. The flow structures in hydrocyclone flow field, including locus of vertical velocity (LZVV), short-cut flow and secondary vortexes, were also investigated and visualized. Moreover, an index matching method was implemented to improve the measurement accuracy.

\section{Experimental}

\subsection{Hydrocyclone}

A $\Phi 35$ mm optical-grade fused quartz (JGS2) (refractive index 1.47) mini-hydrocyclone with a $6^{\circ}$ cone designed for solid-liquid separation was designed [28]. Detailed dimension parameters are shown in Fig. 1 and Table 3. The measured area was approximately $138 \mathrm{~mm}$ in length from top down.

Fig. 1. Dimensions and Coordinates of the mini-hydrocyclone

\section{Table 3. Dimensions of the hydrocyclone}

2.2. Experimental setup and instrumentation

Fig 2. Experimental procedure of $\mathrm{V3V}$ measurement on hydrocyclone flow field

The experimental setup of V3V measurement is shown in Fig. 2. The V3V system 
(Model V8100; TSI Inc., USA) was used to measure the 3D3C flow field. The V3V camera and laser were triggered by a synchronizer (Model 610035; TSI Inc., USA). The measured area was illuminated using a double-cavity Nd:Yag laser at a repetition rate of $15 \mathrm{~Hz}$, wavelength of $532 \mathrm{~nm}$, pulse length of $5 \mathrm{~ns}$, and energy of $500 \mathrm{~mJ} /$ pulse. The light emitted was transformed to an ellipsoidal cone and reflected by a mirror to ease the uneven single-particle image caused by Mie scattering and increase the illumination intensity. A refractive index matching method was implemented in this experiment [29]. The working fluid was $53 \mathrm{wt} \%$ sodium iodide. The properties of the working fluid were closer to water with higher density (1.57). The viscosity $\mu$ of the fluid was $1.1 \times 10^{-6} \mathrm{~m}^{2} / \mathrm{s}$.

\subsection{Measurement procedure and principle with parameter setting}

The whole experimental procedure of $\mathrm{V} 3 \mathrm{~V}$ measurement on hydrocyclone flow field is shown in Fig.2. Calibration was performed inside the acrylic box where the hydrocyclone was temporarily removed. A fluorescent plastic target was traversed with dots spaced at $5 \mathrm{~mm}$ in the horizontal $(x)$ and vertical $(y)$ directions and $2.5 \mathrm{~mm}$ in the depth direction $(z)$. The dot images were simultaneously captured using three CCD cameras at regular $5 \mathrm{~mm}$ intervals starting from the reference plane, where the three views overlapped. During the traversing of target from the reference plane to the defocused planes, the three images of each defocused plane formed a unique triangle. For each triangle, size was referred to the $z$ direction of the plane, and the centroid position was referred to the $x$ and $y$ directions. The calibration considerably eliminated the deviation of the actual camera from a perfect pinhole camera and other distortions caused by the wall condition of measured flow facilities. 
The hydrocyclone model was then fixed at the calibrated space. With perfect refractive index matching, the calibration data remained valid, and the solid wall did not distort the triangles in the particle images. When the flow inside the hydrocyclone reached a pseudo-steady state after 5-10 minutes and the air core was operated to minimum, the particle images of the flow inside hydrocyclone were captured and calculated. The fluorescent particles with particle size of $100 \mu \mathrm{m}$ and density of 1.51 were selected as seeded tracers to increase the light contrast and decrease wall reflection. Three $545 \mathrm{~mm}$ filters were mounted in front of all lenses of the V3V system, which allowed the passage of the light with wavelength above $545 \mathrm{~mm}$. The particles were illuminated by laser and fluoresced. The induced light signal was finally captured by the CCD camera, whereas the light emitted from the laser was thoroughly filtered. Thus, the reflection in the vicinity of wall and the interference from impurities were eliminated.

The four-step image processing using Insight V3V (Ver. 4G, TSI Inc., USA) was as follows:

1. 2D particle identification. All particles were recognized during the image processing of V3V. The global intensity threshold that ranged from 0 to 4096 gray scales was set to 35 , and an overlap value was $50 \%$. The $2 \mathrm{D}$ image of an individual particle was fitted with a Gaussian algorithm. The local peak represented the centroid of the specific particle.

2. 3D particle identification: The three images from each of the camera apertures were combined to determine the $3 \mathrm{D}$ location of the particles. When the three images recognized in the $2 \mathrm{D}$ identification step were overlaid, the correspondent particles in all 
three images were matched to obtain a 3D particle image. A particle in the top image was considered, and the triangle pattern that was predetermined by the calibration was searched.

3. 3D particle tracking: A particle tracking method was applied to obtain the 3D velocity vectors after the $3 \mathrm{D}$ particle locations were obtained in successive image pair (frames $\mathrm{A}$ and $\mathrm{B}$ with reconstructed $3 \mathrm{D}$ particle position). A robust and efficient relaxation method was used for the tracking process. In each interrogation region where five to eight particles were included, the particles tended to move along the same direction.

4. Grid interpolation: A randomly distributed vector map was created during the 3D particle tracking process. Regular Gaussian-weighted interpolation was applied for enhanced display and more convenient post-processing. We created the grid velocity in an ensemble method to improve the spatial resolution of the $\mathrm{V} 3 \mathrm{~V}$ measurement.

In the last step, a template with regular hexahedral grids was used to follow the cylinder-cone shape of the hydrocyclone. Then we interpolated from the measurement result to the template.

\section{Results and discussion}

Ensemble-averaged plot was obtained from 500 measurements of the hydrocyclone velocity field. The Spatial resolution was $2 \mathrm{~mm}$, while in previous V3V measurements the spatial resolution was improve from $5 \mathrm{~mm}$ to $2 \mathrm{~mm}$ [23-24, 30-33].The velocity field was originally generated in a Cartesian coordinate system, but the velocity components were 
calculated in the cylindrical coordinate system. Moreover, one longitudinal section $(\mathrm{C} 0$ : $\theta=$ $\left.0-180^{\circ}\right)$ and seven major vertical cross - sections $(\mathrm{C} 1-\mathrm{C} 6$, with $\mathrm{z}=10,16,50,80,110$, and $120 \mathrm{~mm}$, respectively) were selected to display the measurement result better. Cross-section C2 was just below the tip of the vortex finder, and C3 connected the cylindrical and cone sections. Details of the coordinate system and cross-sections are shown in Fig. 1.

3.1 Three dimensional three component velocity field in hydrocyclone

Fig. 3. Radial velocity distribution on $\mathrm{C} 0, \mathrm{C} 2, \mathrm{C} 4, \mathrm{C} 6$

Radial velocity is the most difficult to measure among the three velocity components. In LDV/PDPA or PIV measurements, if the measured points or plane does not strictly align with the vortex core, the radial velocity is adversely affected by the other velocity components. Fig. 3 presents the radial velocity distribution measured with $\mathrm{V} 3 \mathrm{~V}$. The negative value represents the inward radial velocity. $\mathrm{C} 0, \mathrm{C} 2, \mathrm{C} 4$ and $\mathrm{C} 6$ were extracted to provide a comprehensive display of the 3D radial velocity distribution. The radial velocity was inward under the vortex finder tip, representing the fluid that passed through the vortex finder to discharge as overflow. Inward and outward radial flows occurred in the cross sections of the lower levels.

The radial velocity in r-z plane was not only asymmetric but also displayed a double helix shaped distribution. An exclusively inward velocity occupied the central area of the cross-section. The inward radial velocity was extended to the annular space between vortex finder and hydrocyclone wall, indicating the existence of short-cut flow. The regions below C1 showed alternate inward and outward flows, both in the longitudinal and axial directions. This phenomenon demonstrates that the radial velocity in $\mathrm{r}-\mathrm{z}$ plane is not axisymmetric, at 
least in traditional single-inlet hydrocyclone. The non-asymmetry nature of the radial velocity in r-z plane renders any measurment methods for radial velocity unacceptable. In r-z plane, The highest radial velocity in the selected cross-sections was 0.73 inward (C2), where located the shortcut flow. In other selected cross-sections, the highest radial velocity in r-z plane is $0.63 \mathrm{~m} / \mathrm{s}$ inward (C6) and $0.51 \mathrm{~m} / \mathrm{s}$ outward $(\mathrm{C} 4)$.

Fig. 4. Axial velocity distribution $\mathrm{C} 0, \mathrm{C} 1, \mathrm{C3}, \mathrm{C5}$

Axial velocity, especially the zero axial velocity, is regarded as a reference for separation or classification. Fig. 4 presents the axial velocity distribution measured with V3V. The negative value represents downward axial velocity. $\mathrm{C} 0, \mathrm{C} 1, \mathrm{C} 3$ and $\mathrm{C} 5$ were extracted to provide a comprehensive display of the 3D axial velocity distribution. The axial velocity was positive at the center near the axis of the hydrocyclone, which indicates upward flow. The velocity was negative in the area near hydrocyclone wall, which indicates downward flow. The famous locus of zero vertical velocity (LZVV) existed between the upward and downward flows. The axial velocity distributions showed similar tendencies throughout the entire measured section of the hydrocyclone. The maximum downward axial velocity was located in the hydrocyclone wall area. The magnitude of the downward axial velocity decreased from the wall to the axis, and the velocity then changed upward and increased to reach its maximum at the center of the hydrocyclone. The axial velocity had two peaks close to the central axis as a result of the influence of the air core. Liu [12] also found this phenomenon in the flow measurement of gas cyclone. The highest upward axial velocity $(2.31$ $\mathrm{m} / \mathrm{s}$ ) was located inside the vortex finder. The highest downward axial velocity was located 
near the hydrocyclone wall in the cylinder section. Two counter flows existed in the annular space between the vortex finder and hydrocyclone wall. This phenomenon shows that a secondary vortex was located in this area, and the circulation flows were not only located in the cylinder and cone sections. The asymmetry of the axial velocity was not as severe as that of radial velocity. In practice, the axisymmetry hypothesis can still be adopted. The highest axial velocity in the selected cross-sections is $2.16 \mathrm{~m} / \mathrm{s}(\mathrm{C} 1)$ upward, where is the overflow. In other selected cross-sections, the highest axial velocity is $0.95 \mathrm{~m} / \mathrm{s}(\mathrm{C} 4)$ in the central area.

Fig. 5. Tangential velocity distribution on $\mathrm{C} 0, \mathrm{C1}, \mathrm{C3}, \mathrm{C5}$

Tangential velocity profile is always considered in studies because it generates the centrifugal force for dispersed particles which dominates the separation. This velocity has been thoroughly studied $[17,34]$. Fig. 5 presents the tangential velocity distribution measured with V3V. C0, C1, C3 and C5 were extracted to provide a comprehensive display of the 3D tangential velocity distribution. The positive value indicated a clockwise flow. The axisymmetry hypothesis can still be adopted in the tangential velocity. In the column of the hydrocyclone, the tangential velocity magnitude showed initial decreasing tendency from wall to axis before reaching the minimum. The magnitude then increased from minimum to maximum in the region near the axis. Afterward, the tangential velocity again dropped dramatically to zero. The tangential velocity showed a combination of free vortex in the outer swirl flow and force vortex in the inner swirl flow. C3 represented the connection of the cylinder and cone section. Below this section, the inlet injection had a slight influence on the tangential flow, and the tangential velocity behaved much more like a free vortex. The 
following equation can be used to describe the quasi-free vortex:

$$
v_{\theta} r^{\mathrm{n}}=c
$$

Where, $v_{\theta}$ represents the tangential velocity, $r$ represents the radius, $\mathrm{n}$ is the exponent, and $\mathrm{c}$ is a constant. In this measurement, the parameter $\mathrm{n}$ is fitted to $0.5-0.7$, which is a variable along the axial direction.

The tangential velocity was the highest component among the three velocity components of the flow field inside the hydrocyclone. It reached up to $3.1 \mathrm{~m} / \mathrm{s}$ near the central axis in the cone section and near the upper wall (on the selected cross-sections). Results showed the magnitude of maximum tangential, axial, radial velocity component in the cylindrical and cone part is about $4: 2: 1$.

\subsection{Three dimensional locus of zero vertical velocity}

The locus of zero vertical velocity (LZVV) is an important indicator for separation. Previous researches regarded the area between the two LZVV lines as a low efficiency area, and it was near the LZVV line that particle concentration reached maximum [9]. Thus, the determination of the position of LZVV will help researchers to understand the separation mechanism. The LZVV that constructed in three dimensional is shown in Fig. 6. It can be seen that the LZVV resembles a twisted cone. No zero axial velocity existed in part of the annular space between the vortex finder and hydrocyclone wall, where the axial velocity in this area was exclusively downward. LZVV in the measured hydrocyclone is roughly $0.3 \mathrm{D}$ away from the hydrocyclone wall. According to Dai's report [9], 49\% of the inner space of 
hydrocyclone is low efficiency area.

Fig. 6. Locus of zero vertical velocity

\subsection{Short-cut flow rate}

The shortcut flow directly goes around the outside wall of the vortex finder and enters the overflow over the bottom of the vortex finder. The short cut flow greatly influence the separation efficiency in that some fine particles will be go with the overflow rather than discharge with the underflow. Qian [35] and Fan [21] presented a calculation method of short-cut flow rate: it equals the difference between the inlet flow rate and the downward flow rate. Thus, the short-cut flow rate could be calculated using Equation 2.

$$
L=\int_{\mathrm{r} 1}^{\mathrm{r} 2} 2 \pi r v_{a} d r
$$

Previous researches adopted the axisymmetric hypothesis, they calculate the shortcut flow using 2D data. Since V3V measurement presents three dimensional velocity field data, the integration could be conducted on the area just at the bottom of vortex ring. The outcome of shortcut flow is calculated to be $13 \%$.

\subsection{Downward progression of secondary vortex}

Secondary vortexes are another important structure in hydrocyclone flow field. In some reports, they are recognized as "mantles" [7, 34] or "circulation flow" [19]. They thought the secondary vortexes might hinder the outward transportation of the particles. Fig. 7 shows the surface streamlines on six longitudinal sections with $30^{\circ}$ intervals. The existence of the downward progression of secondary vortex could be seen throughout the whole length of the 
hydrocyclone. Fig. 7(a), (b) and (c) shows the surface streamlines in the annular space between the vortex finder and hydrocyclone wall, cylindrical section, and cone section, separately. It could be found the secondary vortex formed immediately when the flow was injected into the cylinder section. Throughout the entire length of the hydrocyclone flow field, secondary vortices went from the vicinity of the inlet to the bottom of the measured hydrocyclone body. The inheritance relationship of the secondary vortices along a helical line was apparent. Double secondary vortex was found in some areas in the cylindrical and up-cone sections, which indicates that secondary vortices are unstable and may be split into two under some circumstances (with turbulence). The existence of secondary causes great non-axisymmetric of the radial velocity, which makes measurement of this component rather difficult.

\section{Fig.7. Downward progression of secondary vortexes}

\section{Conclusions}

The 3D3C flow field inside a hydrocyclone was measured using a procedure developed based on $\mathrm{V} 3 \mathrm{~V}$ technique. The spatial resolution was enhanced to $2 \mathrm{~mm}$ with improved image quality using a refractive index matching method. The LZVV was constructed in three dimensional and the shortcut flow rate was measured. Results show radial velocity was found to be non-axisymmetric, while the quasi-axisymmetry hypothesis is acceptable for tangential and radial velocity components because the asymmetry is slight and negligible in cylindrical and cone part. The asymmetry of the radial velocity is found to be the consequence of the existing downward progression of secondary vortices. Throughout the entire length of 
hydrocylone, the secondary vortices flowed from the vicinity of the inlet to the bottom of the measured hydrocyclone body. Measurement results show the magnitude of maximum tangential, axial, radial velocity component in $\mathrm{r}-\mathrm{z}$ plane of the cylindrical and cone part is about $4: 2: 1$. The parameter $n$ of tangential velocity in the free vortex area is fitted to be $0.5-0.7$.

\section{Acknowledgments}

We would like to thank for the sponsorship of National Science Foundation for Distinguished Young Scholars of China (Grant No.51125032). 


\section{References}

[1] T.H. Neesse, J. Dueck, H. Schwemmer, M. Farghaly, Using a high pressure hydrocyclone for solids classification in the submicron range, Miner. Eng. 71 (2015) 85-88.

[2] Z.S. Bai, H.L. Wang, S.T. Tu, Oil-water separation using hydrocyclones enhanced by air bubbles, Chem. Eng. Res. Des. 89 (2011) 55-59.

[3] J. Bayo, J. Lopez-Castellanos, R. Martinez-Garcia, A. Alcolea, C. Lardin, Hydrocyclone as a cleaning device for anaerobic sludge digesters in a wastewater treatment plant, J. Clean. Prod. 87 (2015) 550-557.

[4] J. Bergstrom, H. Vomhoff, Experimental hydrocyclone flow field studies, Sep. Purif. Technol. 53 (2007) 8-20.

[5] D. Kelsall, A study of the motion of solid particles in a hydraulic cyclone, Chem. Eng. Res. Des. 30 (1952) 87-108.

[6] S.R. Knowles, D.R. Woods, I.A. Feuerstein, The velocity distribution within a hydrocyclone operating without an air core, Can. J. Chem. Eng. 51 (1973) 263-271.

[7] D. Dabir, Mean velocity measurements in a 3 inches hydrocyclone using laser doppler anemometry, in, Ph. D Thesis, Department of Chemical Engineering, Michigan State University, USA, 1983.

[8] Q. Luo, C.L. Deng, J.R. Xu, L.X. Yu, G.A. Xiong, Comparison of the performance of water-sealed and commercial hydrocyclones, Int. J. Miner. Process. 25 (1989) 297-310.

[9] G.Q. Dai, W.M. Chen, J.M. Li, L.Y. Chu, Experimental study of solid-liquid two-phase flow in a hydrocyclone, Chem. Eng. J. 74 (1999) 211-216.

[10] L.Y. Chu, W.M. Chen, X.Z. Lee, Effects of geometric and operating parameters and feed characters on the motion of solid particles in hydrocyclones, Sep. Purif. Technol. 26 (2002) 237-246.

[11] M.J. Fisher, R.D. Flack, Velocity distributions in a hydrocyclone separator, Exp. Fluids. 32 (2002) 302-312.

[12] Z.L Liu, Y. Zheng, L.F. Jia, J.Y. Jiao, Q.K. Zhang, Stereoscopic PIV studies on the swirling flow structure in a gas cyclone, Chem. Eng. Sci. 61 (2006) 4252-4261.

[13] Z.S. Bai, H.L. Wang, S.T. Tu, Study of Air-Liquid Flow Patterns in Hydrocyclone Enhanced by Air Bubbles, Chem. Eng. Technol. 32 (2009) 55-63. 
[14] L.P.M. Marins, D.G. Duarte, J.B.R. Loureiro, C.A.C. Moraes, A.P.S. Freire, LDA and PIV characterization of the flow in a hydrocyclone without an air-core, J. Pet. Sci. Eng. 70 (2010) 168-176.

[15] N.Y. Zhou, Y.X. Gao, W. An, M.Yang, Investigation of velocity field and oil distribution in an oil-water hydrocyclone using a particle dynamics analyzer, Chem. Eng. J. 157 (2010) 73-79.

[16] Q. Yang, H.L. Wang, J.G. Wang, Z.M. Li, Y. Liu, The coordinated relationship between vortex finder parameters and performance of hydrocyclones for separating light dispersed phase, Sep. Purif. Technol. 79 (2011) 310-320.

[17] Y.H. Zhang, P. Qian, Y. Liu, H.L. Wang, Experimental Study of Hydrocyclone Flow Field with Different Feed Concentration, Ind. Eng. Chem. Res., 50 (2011) 8176-8184.

[18] Z.B. Wang, Y. Ma, Y.H. Jin, Simulation and experiment of flow field in axial-flow hydrocyclone, Chem. Eng. Res. Des. 89 (2011) 603-610.

[19] Y. Liu, Q. Yang, P. Qian, H.L. Wang, Experimental study of circulation flow in a light dispersion hydrocyclone, Sep. Purif. Technol. 137 (2014) 66-73.

[20] B.Y. Cui, D.Z. Wei, S.L. Gao, W.G. Liu, Y.Q. Feng, Numerical and experimental studies of flow field in hydrocyclone with air core, T. Nonferr. Metal. Soc. 24 (2014) 2642-2649.

[21] Y. Fan, J.G. Wang, Z.Y. Bai, J.Y. Wang, H.L. Wang, Experimental investigation of various inlet section angles in mini-hydrocyclones using particle imaging velocimetry, Sep. Purif. Technol. 149 (2015) 156-164.

[22] J.C. Cullivan, R.A. Williams, T. Dyakowski, C.R. Cross, New understanding of a hydrocyclone flow field and separation mechanism from computational fluid dynamics, Miner. Eng. 17 (2004) 651-660.

[23] K.V. Sharp, D. Hill, D.R. Troolin, G. Walters, W. Lai, Volumetric three-component velocimetry measurements of the turbulent flow around a Rushton turbine, Exp. Fluids, 48 (2009) 167-183.

[24] E. Wolf, C.J. Kahler, D.R. Troolin, C. Kykal, W. Lai, Time-resolved volumetric particle tracking velocimetry of large-scale vortex structures from the reattachment region of a laminar separation bubble to the wake, Exp. Fluids, 50 (2011) 977-988.

[25] N. Tounsi, G. Fourrie, H. Oualli, L. Keirsbulck, S. Hanchi, L. Labraga, Volumetric 3-Component Velocimetry Measurements of the Flow Field on the Rear Window of a Generic Car Model, Therm Sci. 16 (2012) 313-320. 
[26] F. Pereira, M. Gharib, D. Dabiri, D. Modarress, Defocusing digital particle image velocimetry: a 3-component 3-dimensional DPIV measurement technique. Application to bubbly flows, Exp. Fluids. 29 (2000) 78-84.

[27] M. Brezinski, K. Saunders, C. Jesser, X. Li, J. Fujimoto, Index Matching to Improve Optical Coherence Tomography Imaging Through Blood, Circulation. 103 (2001) 1999-2003.

[28] I.C. Bicalho, J.L. Mognon, J. Shimoyama, C.H. Ataide, C.R. Duarte, Separation of yeast from alcoholic fermentation in small hydrocyclones, Sep. Purif. Technol. 87 (2012) $62-70$.

[29] D. Amatya, D.R. Troolin, E.K. Longmire, 3D3C Velocity Measurements Downstream of Artificial Heart Valves, Methods. 7 (2009) 9.

[30] D.R. Troolin, E.K. Longmire, Volumetric velocity measurements of vortex rings from inclined exits, Exp. Fluids. 48 (2010) 409-420.

[31] A. Kalmbach, M. Breuer, Experimental PIV/V3V measurements of vortex-induced fluid-structure interaction in turbulentflow-A new benchmark FSI-PfS-2a, J. Fluids Struct. 42 (2013) 369-387.

[32] R. Wahidi, J.P. Hubner, Z. Zhang, 3-D measurements of separated flow over rigid plates with spanwise periodic cambering at low Reynolds number, J. Fluids Struct. 49 (2014) 263-282.

[33] F.C.K. Ting, J. Reimnitz, Volumetric velocity measurements of turbulent coherent structures induced by plunging regular waves, Coastal Eng. 104 (2015) 93-112.

[34] D. Bradley, The hydrocyclone, 1st ed., Pergamon Press, Oxford, New York, 1965.

[35] F.P. Qian, M.Y. Zhang, Effects of the inlet section angle on the flow field of a cyclone, Chem. Eng. Technol. 30 (2007) 1564-1570. 
Figure captions

Fig. 1. Dimensions and Coordinates of the mini-hydrocyclone

Fig. 2. Experimental procedure of $\mathrm{V} 3 \mathrm{~V}$ measurement on hydrocyclone flow field

Fig. 3. Radial velocity distribution on C0, C2, C4, C6

Fig. 4. Axial velocity distribution $\mathrm{C0}, \mathrm{C1}, \mathrm{C3}, \mathrm{C5}$

Fig. 5. Tangential velocity distribution on $\mathrm{C} 0, \mathrm{C} 1, \mathrm{C3}, \mathrm{C} 5$

Fig. 6. Locus of zero vertical velocity

Fig. 7. Downward progression of secondary vortexes 
Table 1. Measurements on the flow field of hydrocyclone

\begin{tabular}{|c|c|c|c|c|}
\hline No. & Title (year) & $\begin{array}{l}\text { Measureme } \\
\text { nt } \\
\text { Technique }\end{array}$ & $\begin{array}{l}\text { Data } \\
\text { Type }\end{array}$ & Main Contents of Measurement \\
\hline 5 & $\begin{array}{l}\text { A study of the motion of solid particles in a hydraulic } \\
\text { cyclone (1952) }\end{array}$ & photography & 1D2C & $\begin{array}{l}\text {-the first to measure the tangential/axial velocity in hydrocyclone } \\
\text {-calculation of radial velocity with material balance method }\end{array}$ \\
\hline 6 & $\begin{array}{l}\text { The velocity distribution within a hydrocyclone operating } \\
\text { without an air core (1973) }\end{array}$ & photography & $1 \mathrm{D} 2 \mathrm{C}$ & $\begin{array}{l}\text {-tangential/axial velocity measurement without an air-core } \\
\text {-check of data for consistency and comparison with the results of earlier } \\
\text { work in hydrocyclones operating with air cores }\end{array}$ \\
\hline 7 & $\begin{array}{l}\text { Velocity measurements in a } 3 \text { inches hydrocyclone using } \\
\text { laser doppler anemometry (1983) }\end{array}$ & LDA & $1 \mathrm{D} 2 \mathrm{C}$ & $\begin{array}{l}\text {-axial/tangential velocity in a hydrocyclone with different vortex finders } \\
\text {-multiple reverse flows in the vortex core }\end{array}$ \\
\hline 8 & $\begin{array}{l}\text { Comparison of the performance of water-sealed and } \\
\text { commercial hydrocyclones (1989) }\end{array}$ & LDA & $1 \mathrm{D} 3 \mathrm{C}$ & $\begin{array}{l}\text {-three-dimensional velocities in both water-sealed and ordinary } \\
\text { hydrocyclones with a LDA }\end{array}$ \\
\hline 9 & $\begin{array}{l}\text { Experimental study of solid-liquid two-phase flow in a } \\
\text { hydrocyclone (1999) }\end{array}$ & PDPA & $1 \mathrm{D} 2 \mathrm{C}$ & $\begin{array}{l}\text {-radial and axial velocity with particle size and concentration } \\
\text {-LZVV as indicator for particle concentration and separation boundary }\end{array}$ \\
\hline 10 & $\begin{array}{l}\text { Effects of geometric and operating parameters and feed } \\
\text { characters on the motion of solid particles in } \\
\text { hydrocyclones (2002) }\end{array}$ & PDA & $1 \mathrm{D} 2 \mathrm{C}$ & $\begin{array}{l}\text {-the effects of geometric (diameter of the underflow pipe) and operating } \\
\text { (inlet pressure, flow rate) parameters and feed characters (particle density, } \\
\text { size and concentration) on the motion of solid particles }\end{array}$ \\
\hline 11 & Velocity distributions in a hydrocyclone separator (2002) & LDV & $1 \mathrm{D} 3 \mathrm{C}$ & $\begin{array}{l}\text {-measurement of three component of velocity in different flow rate and } \\
\text { reject rate }\end{array}$ \\
\hline 12 & $\begin{array}{l}\text { Stereoscopic PIV studies on the swirling flow structure in } \\
\text { a gas cyclone (2006) }\end{array}$ & PIV & $2 \mathrm{D} 3 \mathrm{C}$ & $\begin{array}{l}\text {-measurement of instantaneous tangential, axial, and radial velocities } \\
\text { simultaneously using Stereo-PIV in a cyclone }\end{array}$ \\
\hline 13 & $\begin{array}{l}\text { Study of Air-Liquid Flow Patterns in Hydrocyclone } \\
\text { Enhanced by Air Bubbles (2009) }\end{array}$ & PDPA & $1 \mathrm{D} 2 \mathrm{C}$ & $\begin{array}{l}\text {-the measurement of velocities of air and liquid phases in a hydrocyclone } \\
\text { enhanced by air bubbles using PDPA }\end{array}$ \\
\hline 14 & $\begin{array}{l}\text { LDA and PIV characterization of the flow in a } \\
\text { hydrocyclone without an air-core (2010) }\end{array}$ & LDA/PIV & $\begin{array}{l}1 \mathrm{D} 2 \mathrm{C} / \\
2 \mathrm{D} 3 \mathrm{C}\end{array}$ & $\begin{array}{l}\text {-tangential/axial velocity with PIV/LDV and radial velocity with PIV } \\
\text {-accuracy of the present data (enough to serve as reference data for the } \\
\text { validation of numerical simulations) }\end{array}$ \\
\hline 15 & $\begin{array}{l}\text { Investigation of velocity field and oil distribution in an } \\
\text { oil-water hydrocyclone using a particle dynamics analyzer }\end{array}$ & PDA & $1 \mathrm{D} 2 \mathrm{C}$ & $\begin{array}{l}\text {-distribution of velocity field and oil droplets in a } 50 \mathrm{~mm} \text { oil-water } \\
\text { separation glass hydrocyclone }\end{array}$ \\
\hline 16 & $\begin{array}{l}\text { The coordinated relationship between vortex finder } \\
\text { parameters and performance of hydrocyclones for } \\
\text { separating light dispersed phase (2011) }\end{array}$ & PDPA & $1 \mathrm{D} 2 \mathrm{C}$ & $\begin{array}{l}\text {-influence of change of structure and size of vortex finder on zero axial } \\
\text { velocity wave zone (ZAVWZ) } \\
\text {-tangential velocity gradient, centrifugal separation factor and size } \\
\text { distribution of dispersed phase. }\end{array}$ \\
\hline 17 & $\begin{array}{l}\text { Experimental Study of Hydrocyclone Flow Field with } \\
\text { Different Feed Concentration (2011) }\end{array}$ & PDPA & $1 \mathrm{D} 2 \mathrm{C}$ & $\begin{array}{l}\text { - influence of feed concentration on tangential/axial velocities, turbulence, } \\
\text { dimension of circular flow and zero velocity value in hydrocyclone }\end{array}$ \\
\hline 18 & $\begin{array}{l}\text { Simulation and experiment of flow field in axial-flow } \\
\text { hydrocyclone (2011) }\end{array}$ & LDV & $1 \mathrm{D} 2 \mathrm{C}$ & $\begin{array}{l}\text {-verification for the numerical results of the tangential and axial velocities } \\
\text { inside an axial-flow hydrocyclone with LDV }\end{array}$ \\
\hline 19 & $\begin{array}{l}\text { Experimental study of circulation flow in a light } \\
\text { dispersion hydrocyclone (2014) }\end{array}$ & PDPA & $1 \mathrm{D} 2 \mathrm{C}$ & -axial velocity field in hydrocyclones with various vortex finders lengths \\
\hline 20 & $\begin{array}{l}\text { Numerical and experimental studies of flow field in } \\
\text { hydrocyclone with air core (2014) }\end{array}$ & PIV & $2 \mathrm{D} 2 \mathrm{C}$ & $\begin{array}{l}\text { - construction of velocity vector map over a vertical plane crossing the } \\
\text { vertical axis in the hydrocyclone }\end{array}$ \\
\hline 21 & $\begin{array}{l}\text { Experimental investigation of various inlet section angles } \\
\text { in mini-hydrocyclones using particle imaging velocimetry } \\
\text { (2015) }\end{array}$ & PIV & $2 \mathrm{D} 2 \mathrm{C}$ & $\begin{array}{l}\text { - axial and radial velocity in hydrocyclones with different inlet angles } \\
\text { - construction of streamline diagrams calculated from the vectors } \\
\text { - calculation of the shortcut flow rate }\end{array}$ \\
\hline
\end{tabular}


Table. 2. Comparison of the three optical measurement methods

Instruments

LDV/PDPA/3D PDPA

Classic PIV/Stero-PIV

V3V

Measured Area

Single point

Surface

Volume

Dimensions and Components

1D2C/1D2C /1D3C

2D2C /2D3C

3D3C 
Table.3. Dimensions of the hydrocyclone

\begin{tabular}{ccccccccc}
\hline $\mathrm{D} / \mathrm{mm}$ & $\mathrm{L}_{1} / \mathrm{D}$ & $\mathrm{L}_{2} / \mathrm{D}$ & $\mathrm{d}_{\mathrm{o}} / \mathrm{D}$ & $\mathrm{D}_{\mathrm{o}} / \mathrm{D}$ & $\mathrm{d}_{\mathrm{p}} / \mathrm{D}$ & $\mathrm{d}_{\mathrm{u}} / \mathrm{D}$ & $\mathrm{d} / \mathrm{mm}$ & $\mathrm{h} / \mathrm{mm}$ \\
\hline 35 & 1.48 & 8.78 & 0.24 & 0.68 & 0.57 & 0.08 & 5.6 & 8.4 \\
\hline
\end{tabular}




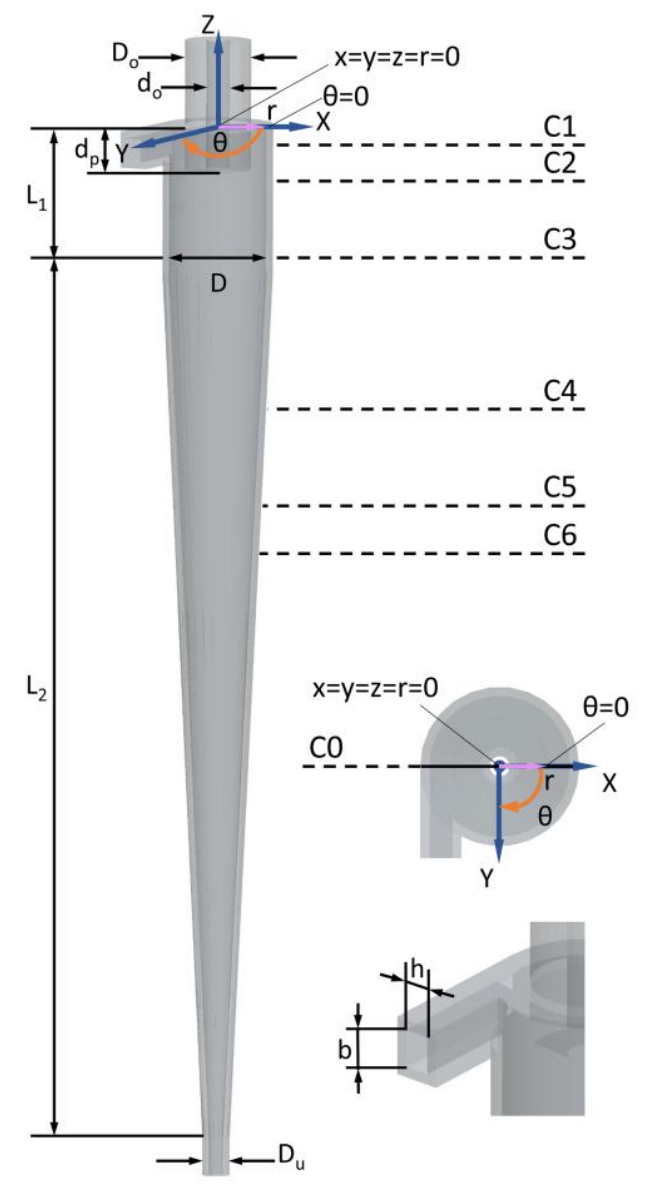




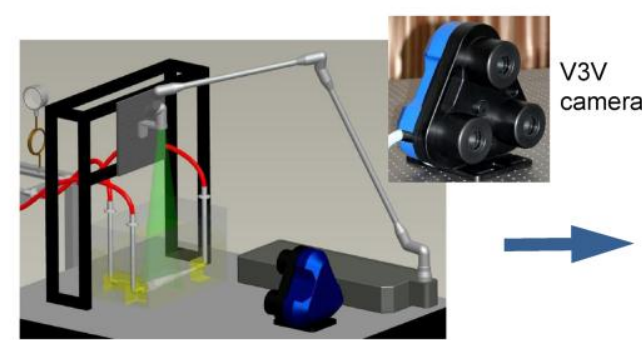

Setup of V3V camera and light path

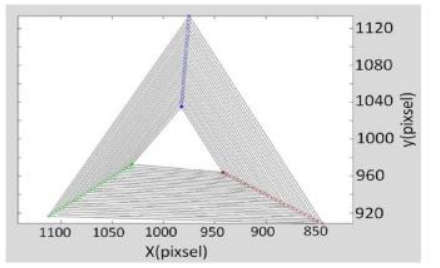

Calibration of the $3 \mathrm{D}$ position

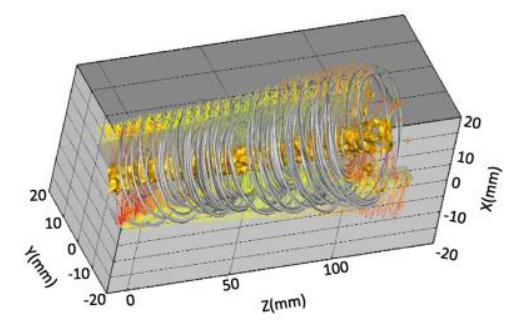

Calculation of velocity vector and flow field visualization

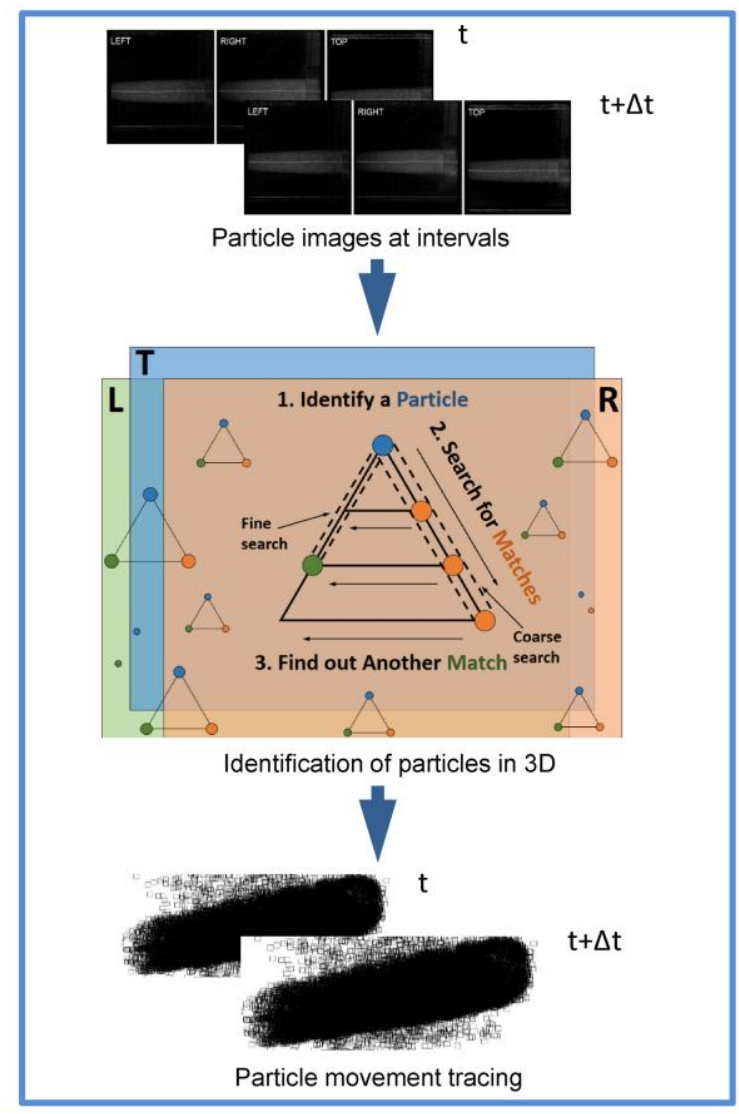




\begin{tabular}{|c|}
\hline Radial Velocity \\
\hline 0.30 \\
0.27 \\
0.24 \\
0.21 \\
0.17 \\
0.14 \\
0.11 \\
0.08 \\
0.05 \\
0.02 \\
-0.02 \\
-0.05 \\
-0.08 \\
-0.11 \\
-0.14 \\
-0.17 \\
-0.21 \\
-0.24 \\
-0.27 \\
-0.30 \\
\hline
\end{tabular}

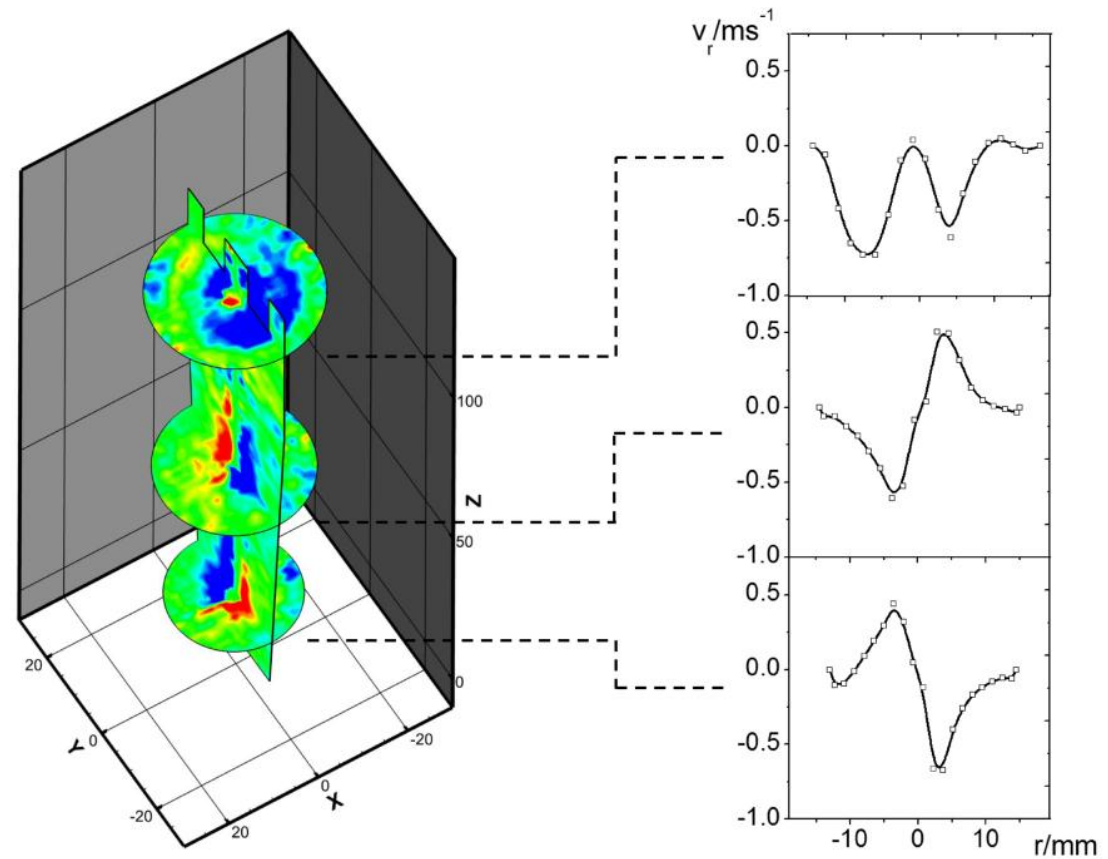




\begin{tabular}{|c|}
\hline \multicolumn{2}{|c|}{ Axial Velocity } \\
\hline 1.00 \\
0.89 \\
0.79 \\
0.68 \\
0.58 \\
0.47 \\
0.37 \\
0.26 \\
0.16 \\
0.05 \\
-0.05 \\
-0.16 \\
-0.26 \\
-0.37 \\
-0.47 \\
-0.58 \\
-0.68 \\
-0.79 \\
-0.89 \\
-1.00 \\
\hline
\end{tabular}
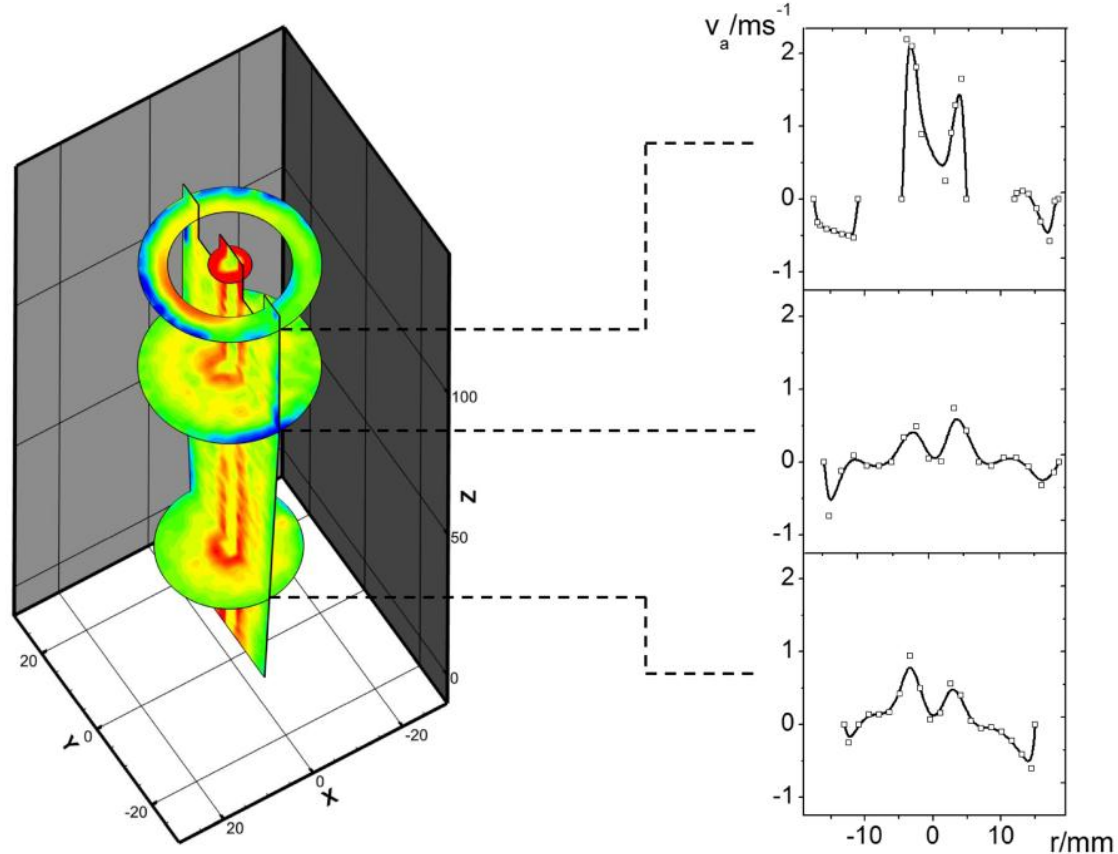


\begin{tabular}{|c|}
\hline \multicolumn{1}{|c|}{ Tangential Velocity } \\
\hline 3.60 \\
\hline 3.41 \\
3.22 \\
\hline 3.03 \\
\hline 2.84 \\
\hline 2.65 \\
\hline 2.46 \\
\hline 2.27 \\
\hline 2.08 \\
\hline 1.89 \\
\hline 1.71 \\
\hline 1.52 \\
\hline 1.33 \\
\hline 1.14 \\
\hline 0.95 \\
\hline 0.76 \\
\hline 0.57 \\
\hline 0.38 \\
\hline 0.19 \\
\hline 0.00 \\
\hline
\end{tabular}

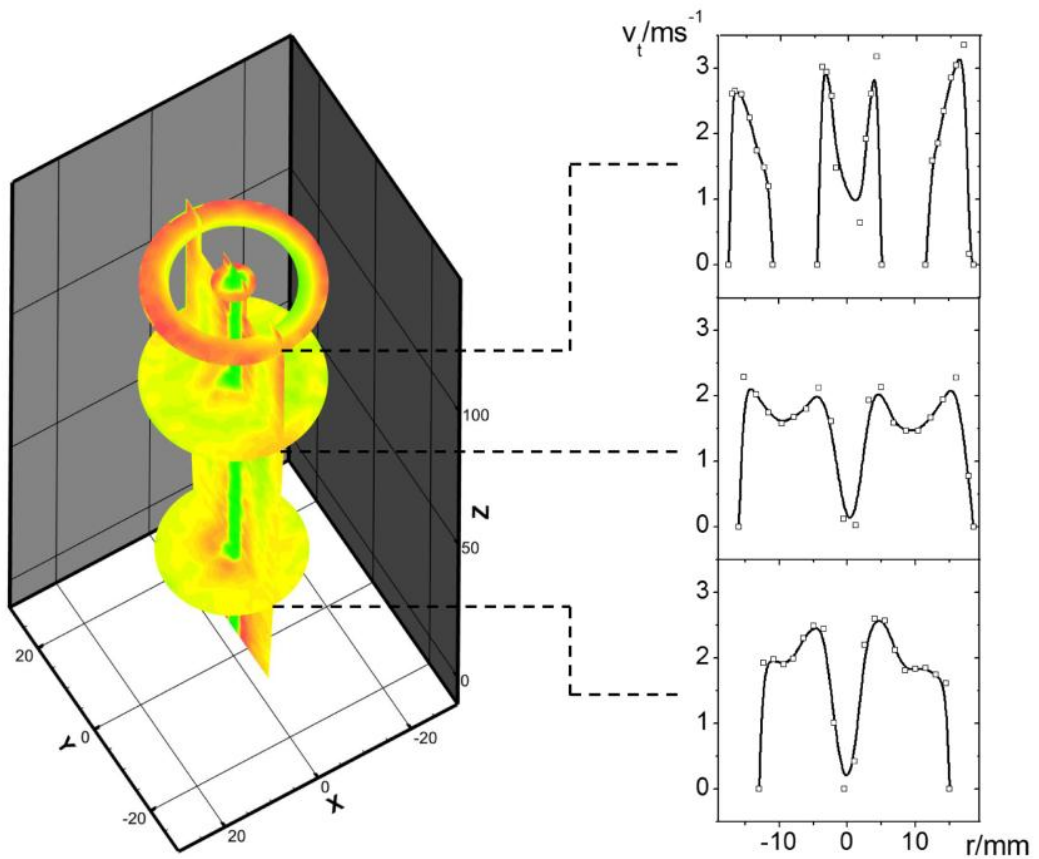




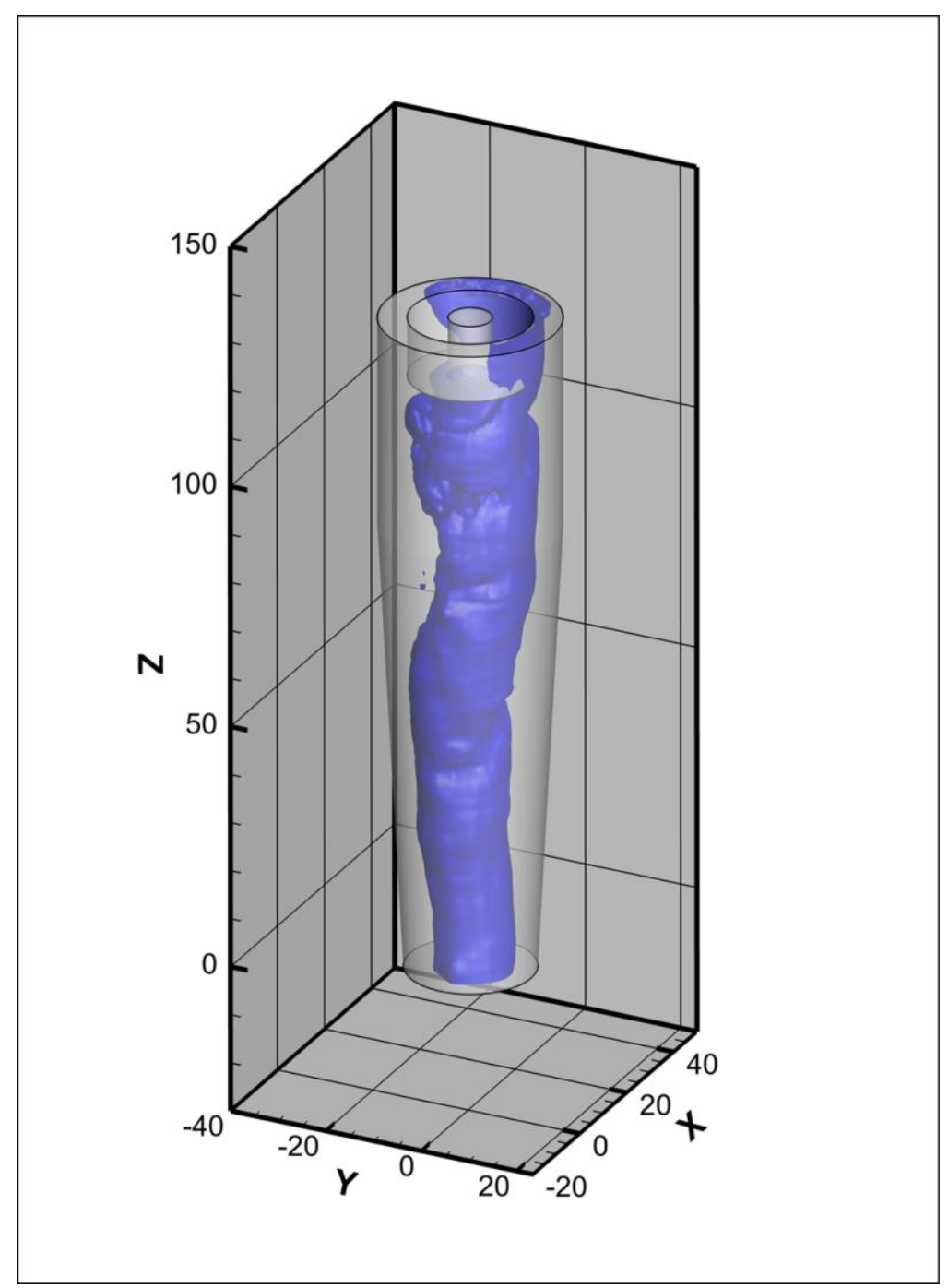


(a)

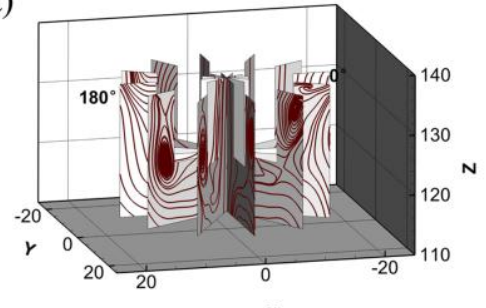

(b)

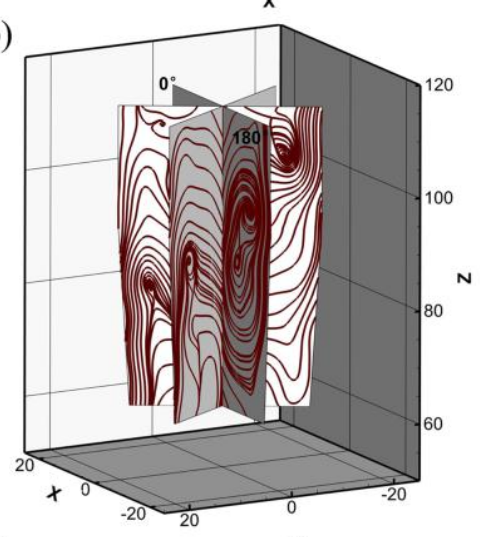

(c)

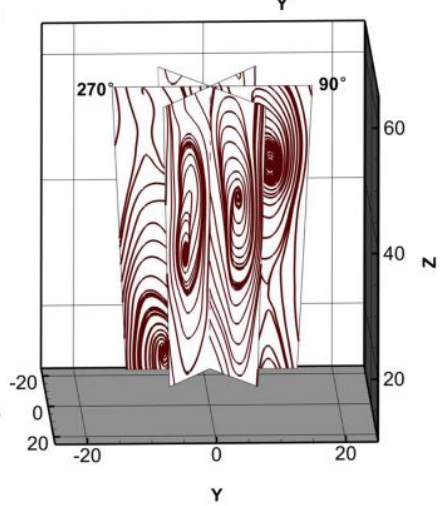

